\title{
The Combined Treatment of PTSD with Borderline Personality Disorder
}

\section{Melanie S. Harned, PhD}

\author{
Address \\ Behavioral Research and Therapy Clinics, Department of Psychology, University of \\ Washington, Box 355915, Seattle, WA 98195-5915, USA \\ Email: mharned@uw.edu
}

Published online: 20 September 2014

C Springer International Publishing AG 2014

Keywords Borderline personality disorder · PTSD · Intentional self-injury · Psychotherapy

\section{Opinion statement}

Psychotherapies are currently the front-line approach to treating individuals with comorbid post-traumatic stress disorder (PTSD) and borderline personality disorder (BPD). Of the available psychotherapeutic treatment options, those that have been evaluated in randomized controlled trials (RCTs) and shown to be efficacious in reducing PTSD among individuals with BPD include: Prolonged Exposure (PE), Cognitive Processing Therapy (CPT), Dialectical Behavior Therapy (DBT), DBT for PTSD (DBT-PTSD), and DBT with the DBT Prolonged Exposure protocol (DBT + DBT PE). In addition, Narrative Exposure Therapy (NET) has shown preliminary evidence of effectiveness in an open trial. The available research indicates that individuals with a primary diagnosis of PTSD and mild or subthreshold BPD (e.g., without suicidal or serious self-injurious behavior or other severe comorbidities) can be effectively treated via brief (9-14 session), single-diagnosis treatments such as PE and CPT that focus solely on treating PTSD. PE and CPT appear to be comparably efficacious for individuals with PTSD and mild BPD. Individuals with PTSD and a moderate level of BPD (e.g., active non-suicidal self-injury [NSSI] without recent lifethreatening behavior, some significant comorbidities) have shown good outcomes in DBTPTSD, a 12-week, residential, phase-based treatment that targets comorbid problems (e.g., emotion dysregulation, psychosocial impairment) before and after addressing PTSD. Finally, individuals with PTSD and a severe level of BPD (e.g., with recent and/or acute suicidal behavior, serious NSSI and multiple severe comorbidities) have been effectively treated in longer-term (one year), integrated treatments such as DBT and DBT + DBT PE that concurrently target BPD, PTSD, and other related problems. Of these integrated treatments, DBT + DBT PE appears to be more efficacious than DBT alone in improving PTSD, suicidal and self-injurious behavior, and other trauma-related outcomes among severe BPD patients. 


\section{Introduction}

Borderline personality disorder (BPD) is a complex and often severe psychological disorder characterized by pervasive emotion dysregulation, impulsive behavior, unstable relationships, and recurrent suicidal and self-injurious behavior. Individuals with BPD exhibit high rates of comorbidity and have been found to meet diagnostic criteria for an average of 3.4 to 4.2 lifetime Axis I disorders [1, 2]. Post-traumatic stress disorder (PTSD) is one of the most common comorbid disorders among individuals with BPD and its core features include intrusive re-experiencing of traumatic events, avoidance of trauma-related thoughts and situations, emotional numbing, and hyperarousal. Among individuals with BPD, rates of comorbid PTSD range from approximately $30 \%$ in community samples $[3,4]$ to $50 \%$ in clinical samples $[5,6]$. Females constitute approximately two-thirds of individuals with both BPD and PTSD in the community [4] and more than $85 \%$ of this comorbid population in clinical settings [6]. Although the high degree of comorbidity between BPD and PTSD has led to some debate about whether BPD may be better conceptualized as a trauma spectrum disorder such as 'complex' PTSD, key differences in the clinical phenomenology and neurobiology of these disorders warrant their conceptualization as distinct, albeit commonly co-occurring disorders [7].

Individuals with both BPD and PTSD tend to be more impaired than those with either disorder alone. Compared with individuals with either BPD or PTSD, those with both diagnoses have higher rates of other Axis I disorders $[4,5,8,9]$, an increased risk of suicide attempts and non-suicidal self-injury (NSSI) [4, 5, 9-
11], earlier onset of abuse and more severe trauma [4, 5, $12,13]$, greater emotion dysregulation [5, 14], more frequent inpatient psychiatric hospitalization [15], poorer physical health $[8,12]$, and more impaired global functioning $[13,15]$. Moreover, the presence of PTSD has been found to decrease the likelihood of attaining diagnostic remission from BPD over 6 and 10 years of naturalistic follow-up $[16,17]$. Taken together, these findings suggest not only that individuals with BPD and PTSD are likely to have a particularly complex and severe presentation, but also that successful treatment of BPD may be contingent upon the resolution of PTSD.

Historically, BPD has often been viewed as a contraindication for trauma-focused treatment due to concerns that such treatment would lead to symptom worsening (e.g., increased suicidality). Over the past decade, however, research has provided convincing evidence that PTSD can be safely and effectively treated among individuals with BPD of varying levels of severity. To date, the available research supports the efficacy of several psychotherapeutic approaches to treating PTSD among individuals with BPD. Although several evidence-based pharmacological treatments for PTSD exist, none have been evaluated specifically for individuals with comorbid PTSD and BPD. Thus, the present review is restricted to psychotherapeutic treatments that have been evaluated in terms of their effect on PTSD among individuals with BPD. These treatments are grouped in terms of their general approach, including single-diagnosis, phase-based, and integrated treatments. Of note, psychotherapies that have included individuals with BPD but have not reported PTSD outcomes specific to this subgroup are not reviewed [e.g., 18-20].

\section{Psychotherapeutic treatment options}

- Single-diagnosis treatments are those that focus primarily or only on treating PTSD. These treatments may include individuals with various comorbid problems such as BPD, but comorbid problems are not targeted directly. Thus, any improvements in comorbid problems that occur during these treatments are a secondary result of treating PTSD. 
Standard procedure

Contraindications

Complications

Special points
PE [21] is a brief outpatient treatment that was originally developed for women with sexual assault-related PTSD and has since been shown to be efficacious in a variety of trauma populations [22]. The primary components of PE include imaginal exposure to trauma memories and in vivo exposure to feared but safe situations. PE is typically delivered in 9-15 weekly or biweekly individual therapy sessions.

Two randomized controlled trials (RCTs) have evaluated the efficacy of PE among individuals with full or partial BPD or BPD characteristics. One RCT [23, Class I] examined the impact of full or partial BPD on treatment outcome following PE, stress inoculation training (SIT, focused on anxiety management skills), and a combined PE/SIT treatment. Participants were female sexual and non-sexual assault survivors with a primary diagnosis of PTSD related to an assault occurring after age 16. Exclusion criteria were: acute suicidality, suicide attempt or serious self-injurious behavior in the past 3 months, ongoing abuse, substance dependence, bipolar disorder, and psychotic disorders. In the treatment completer sample $(n=58), 9(15.5 \%)$ met full or partial criteria for BPD. Given the small sample of BPD patients, analyses were not conducted separately by treatment. When the three treatments were combined, BPD was not significantly related to outcome. However, women with full or partial BPD were significantly less likely than those without BPD to achieve good end-state functioning ( $11 \%$ vs $51 \%$ ), which was defined as being below clinical cut-offs on PTSD, depression, and general anxiety.

A second RCT [24, Class I] evaluated the effect of borderline personality characteristics (BPC) on outcomes following PE or Cognitive Processing Therapy (CPT). Participants included 131 adult female rape victims with PTSD, of whom 39 (25.2\%) were identified as having a clinical level of BPC according to self-report. Women with PTSD related to an index rape occurring in adulthood or childhood that was not perpetrated by a family member were included. Women with serious suicidal intent, recent suicidal or self-injurious behavior, ongoing abuse, current substance dependence, or bipolar or psychotic disorders were excluded. Results indicated that BPC scores were unrelated to treatment dropout and there was no evidence that BPC were related to worse outcomes in PTSD or other trauma-related symptoms. PE and CPT were comparably effective for individuals with BPC.

Individuals with acute suicidality (i.e., ideation with intent and a plan) and recent suicidal or serious self-injurious behavior are excluded from PE. Many studies of PE, including the two RCTs reviewed above, have also excluded individuals with ongoing abuse, substance dependence, bipolar disorder, and psychotic disorders. Of note, emerging research suggests that PE can be safely and effectively applied in several populations previously thought to be inappropriate for this treatment, such as those with substance dependence and psychotic disorders. See [25] for a recent review of contraindications for PE.

None identified.

The existing research on PE for this population has either included individuals meeting partial criteria for BPD [23] or has not assessed the full BPD diagnosis [24]. Further, these studies have excluded women with either an index assault occurring in childhood [23] or an index assault involving incest [24]. Both studies excluded individuals with many behaviors common in severe BPD (e.g., acute suicidality, recent suicidal and self-injurious behavior, substance 


\section{Cognitive processing therapy (CPT)}

Standard procedure

Contraindications

Complications

Special points dependence). Thus, additional research is needed to evaluate the efficacy of PE for individuals meeting full criteria for BPD, exhibiting more severe levels of BPD, and with childhood assault or incest-related PTSD. Finally, additional treatment may be necessary for BPD patients who do not achieve good endstate functioning after a standard course of PE.

CPT [26] is a brief outpatient treatment for PTSD that was originally developed to treat sexual assault-related PTSD and has since been found to be efficacious for a variety of trauma types [27]. CPT consists of 12 weekly or biweekly sessions that can be delivered in individual, group, or a combined format. The treatment is primarily cognitive and focuses largely on identifying and challenging trauma-related beliefs. It can also include an exposure component in the form of writing a detailed account of the traumatic event.

To date, one RCT [24, Class I; reviewed in detail above] has evaluated the effect of BPC on outcomes in CPT among adult female rape victims. This study found no effect of borderline characteristics on treatment dropout or PTSD and other trauma-related outcomes, suggesting that CPT is comparably efficacious for individuals with and without BPC.

Individuals with acute suicidality (i.e., ideation with intent and a plan) and recent suicidal or serious self-injurious behavior are excluded from CPT. Other common exclusion criteria include ongoing abuse, substance dependence, bipolar disorder, and current psychosis.

None identified.

The only RCT that has evaluated the efficacy of CPT for this population did not assess for the full BPD diagnosis and excluded women with serious suicidal intent, recent suicidal or self-injurious behavior, ongoing abuse, current substance dependence, bipolar and psychotic disorders, or an index rape involving incest. Thus, the generalizability of these findings to individuals meeting full diagnostic criteria for BPD, exhibiting more severe levels of BPD, and incestrelated PTSD is unknown.

Phase-Based Treatments

- Phase-based treatments typically include an initial phase focused on stabilization and behavioral skills training followed by a second phase of trauma-focused treatment. Some treatments also include a third phase in which impairments in psychosocial functioning are typically addressed.

Dialectical behavior therapy for PTSD (DBT-PTSD) address PTSD related to childhood sexual abuse. DBT-PTSD is delivered as a 12week residential treatment that includes three treatment phases. The first phase (weeks 1-4) focuses on psychoeducation, identification of typical cognitive, emotional, and behavioral strategies to escape emotions, and teaching of DBT skills to control these behaviors. The second phase (weeks 5-10) consists of trauma-focused cognitive and exposure-based interventions. The final phase (weeks 11-12) addresses radical acceptance of trauma-related facts and psychosocial functioning. Patients receive biweekly individual sessions (23 total), 
Contraindications DBT-PTSD is not used with individuals with recent life-threatening behavior (e.g., serious suicide attempts) and some severe comorbidities (substance dependence, schizophrenia, and severe anorexia nervosa).

Complications None identified. There was no evidence of worsening of PTSD, NSSI or suicidality in DBT-PTSD.

Special points Although women who were actively engaging in NSSI were included, treatment occurred in a residential setting that allowed for close monitoring of patient safety. The generalizability of these findings to outpatient samples of BPD patients as well as those with recent life-threatening behavior and the specified exclusionary diagnoses (e.g., substance dependence) is unknown. In addition, DBT-PTSD is primarily intended to target PTSD and associated trauma-related problems, and its effects on BPD symptoms did not differ from those obtained in a non-active treatment control.

- Integrated treatments are intended to comprehensively address the full range of problems with which individuals with PTSD and BPD present. This includes directly targeting both PTSD- and BPD-related problems, as well as factors that may explain the relationships between these two disorders. In addition, other problems (e.g., additional comorbid disorders, psychosocial functioning) are addressed as needed.

DBT [30] is a comprehensive cognitive behavioral therapy that is the most well researched treatment available for BPD. DBT combines change-based interventions from behavior therapy (e.g., skills training, exposure, cognitive modification, contingency management) with acceptance-based interventions derived from Western contemplative and eastern Zen practices (e.g., validation, radical acceptance). DBT was developed for chronically suicidal individuals with BPD and is idiographically applied to address a hierarchy of treatment targets: (i) life-threatening behaviors (e. g., suicide attempts, NSSI), (ii) therapy- 
Contraindications DBT requires patients to achieve control over life-threatening behaviors and serious therapy-interfering behaviors prior to addressing PTSD.

Complications No complications related to targeting PTSD during DBT have been identified. However, several studies have shown that BPD individuals with PTSD exhibit poorer outcomes during DBT in terms of NSSI and suicidality [32, 33]. Thus, untreated/ active PTSD may complicate treatment of BPD-related problems during DBT.

Special points In the two studies reporting PTSD outcomes during DBT, PTSD was either not routinely targeted [1] or direct targeting of PTSD via formal exposure procedures was prohibited $[31 \bullet \bullet]$. Thus, these results are best interpreted as demonstrating the indirect effects of DBT on PTSD when this comorbid disorder is not directly targeted. In addition, individuals with bipolar and psychotic disorders were excluded from both studies and the generalizability of these findings to individuals with those disorders is unknown.

Dialectical behavior therapy with the dbt prolonged exposure protocol $(D B T+D B T P E)$

The DBT PE protocol was developed to facilitate routine and direct targeting of PTSD during standard DBT [34]. The combined DBT + DBT PE treatment includes 1 year of DBT (including all four treatment modes as described above) that begins with a focus on reducing behavioral dyscontrol and increasing behavioral skills. If/when patients meet specified readiness criteria (e.g., abstinence from suicidal and self-injurious behaviors for at least 2 months, no serious therapy-interfering behaviors), the DBT PE protocol is integrated into individual therapy sessions to directly target PTSD. This protocol is based on PE [21] with adaptations made to address the specific needs and characteristics of BPD patients. The primary treatment components include imaginal exposure to trauma memories and in vivo exposure to avoided situations. Following completion of the DBT PE protocol, standard DBT is continued with a focus on addressing any remaining treatment targets (e.g., psychosocial functioning). 
Contraindications In DBT + DBT PE, patients are required to achieve control over life-threatening behaviors and serious therapy-interfering behaviors prior to beginning the DBT PE protocol.

Complications None identified. Of note, adding the DBT PE protocol to DBT is associated with improvement, not worsening, of PTSD and suicidal and self-injurious urges and

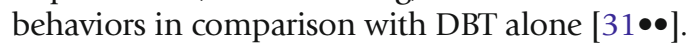

Special points The RCT evaluating DBT + DBT PE used a relatively small sample and replication in a large-scale RCT is needed. Individuals with bipolar and psychotic disorders were excluded from the open trial and RCT and additional research is needed to evaluate the generalizability of these findings to BPD patients with these diagnoses.

Emerging therapies

- The following psychotherapy has been evaluated in a preliminary open trial, and an RCT is complete but not yet published.

Narrative exposure therapy (NET)

NET is a brief treatment for PTSD that is designed for survivors of multiple and complex traumas such as victims of organized violence and conflict [37]. NET is typically delivered in 5-10 weekly or biweekly individual sessions. The primary component of NET is the creation of a written, cohesive life narrative that includes significant traumatic and non-traumatic events.

NET has been evaluated in an open feasibility trial with ten adult women with BPD and PTSD [38, Class IV]. Women who were actively engaging in NSSI were included, but those with acute suicidality, a recent suicide attempt, and other severe comorbidities (e.g., substance dependence, psychosis, body mass index $<18$ ) were excluded. Treatment lasted an average of 14 sessions and was primarily delivered in an inpatient setting, although three women were treated solely on an outpatient basis. Results indicated large and significant improvements from pre-treatment to 6 months after therapy in PTSD, depression, and dissociation, but not BPD symptoms. 
Contraindications In this study, NET was not provided to individuals with recent suicidal behavior, acute suicidality, and other severe comorbidities.

Complications None identified.

Special points Although NET was delivered to women who were actively engaging in NSSI, treatment occurred primarily in an inpatient setting where patient safety could be closely monitored. The generalizability of these findings to outpatient samples of BPD patients as well as those with significant suicidality and other severe comorbidities is unknown. In addition, NET is intended to treat PTSD and does not appear to improve BPD symptoms.

\section{Conclusion}

- To date, no psychotherapies have been evaluated as a treatment for PTSD among children or adolescents with BPD or BPD traits. However, several of the treatments reviewed above have been adapted for adolescents and/ or children and evaluated in RCTs. Specifically, developmentally adapted PE has been shown to be superior to time-limited dynamic therapy and supportive counseling in improving PTSD and other outcomes among adolescents girls with sexual abuse-related PTSD [39, 40]. Similarly, an adapted version of narrative exposure therapy outperformed a waiting list in reducing PTSD among refugee children and adolescents living in exile [41]. Finally, a shortened form of DBT for adolescents has been found to be more efficacious than enhanced usual care in reducing self-harm, suicidal ideation, and depression among adolescents with repeated suicidal and self-harming behavior [42]. Future research is needed to evaluate the efficacy of these treatments specifically for children and adolescents with comorbid PTSD and BPD or BPD traits.

For decades, common clinical lore has suggested that providing trauma-focused treatment to individuals with BPD is likely to be ineffective at best and potentially lethal at worst. However, due to considerable advances in research over the past decade, there are now a number of psychotherapies that have been shown to be both safe and efficacious for treating PTSD among BPD patients with varying levels of severity. Ideally these findings will not only help to assuage clinicians' concerns, but will also provide hope for individuals suffering from these complex disorders. At the same time, research in this area is still in its infancy and more rigorous and large-scale studies are needed to replicate and extend these findings.

\section{Compliance with Ethics Guidelines}

Conflict of Interest

Melanie S. Harned received funding from the National Institute of Mental Health to develop and evaluate DBT + DBT PE. She is also a trainer and consultant in DBT and DBT + DBT PE for Behavioral Tech LLC. 
Human and Animal Rights and Informed Consent

This article does contain references to studies with human or animal subjects performed by any of the authors. These studies are in refs $[1,5,31 \bullet \bullet, 32,35,36 \bullet]$.

\section{References and Recommended Reading}

Papers of particular interest, published recently, have been

highlighted as:

- Of importance

$\bullet \quad$ Of major importance

1. Harned MS, Chapman AL, Dexter-Mazza ET, Murray A, Comtois KA, Linehan MM. Treating co-occurring Axis I disorders in recurrently suicidal women with borderline personality disorder: a 2-year randomized trial of dialectical behavior therapy versus community treatment by experts. J Consult Clin Psychol. 2008;76(6):1068-75.

2. Zimmerman M, Mattia JI. Axis I diagnostic comorbidity and borderline personality disorder. Compr Psychiatry. 1999;40(4):245-52.

3. Grant BF, Chou SP, Goldstein RB, Huang B, Stinson FS, Saha TD, et al. Prevalence, correlates, disability, and comorbidity of DSM-IV borderline personality disorder: results from the wave $2 \mathrm{Na}$ tional Epidemiologic Survey on Alcohol and Related Conditions. J Clin Psychiatry. 2008;69(4):533-45.

4. Pagura J, Stein MB, Bolton JM, Cox BJ, Grant B, Sareen J. Comorbidity of borderline personality disorder and posttraumatic stress disorder in the U.S. population. J Psychiatr Res. 2010;44(16):1190-8.

5. Harned MS, Rizvi SL, Linehan MM. The impact of cooccurring posttraumatic stress disorder on suicidal women with borderline personality disorder. Am J Psychiatry. 2010;167(10):1210-7.

6. Zanarini MC, Frankenburg FR, Dubo ED, Sickel AE, Trikha A, Levin A, et al. Axis I comorbidity of borderline personality disorder. Am J Psychiatry. 1998;155(12):1733-9.

7. Ford JD, Courtois CA. Complex PTSD, affect dysregulation, and borderline personality disorder. Borderline Personality Disorder and Emotion Dysregulation 2014; 1(9): http://www.bpded.com/content/1-1/9.

8. Bolton EE, Mueser KT, Rosenberg SD. Symptom correlates of posttraumatic stress disorder in clients with borderline personality disorder. Compr Psychiatry. 2006;47(5):357-61.

9. Rusch N, Corrigan PW, Bohus M, Kuhler T, Jacob GA, Lieb K. The impact of posttraumatic stress disorder on dysfunctional implicit and explicit emotions among women with borderline personality disorder. J Nerv Ment Dis. 2007;195(6):537-9.

10. Cougle JR, Keough ME, Riccardi CJ, Sachs-Ericsson N. Anxiety disorders and suicidality in the National Comorbidity Survey-Replication. J Psychiatr Res. 2009;43(9):825-9.

11. Nepon J, Belik SL, Bolton J, Sareen J. The relationship between anxiety disorders and suicide attempts: findings from the National Epidemiologic Survey on Alcohol and Related Conditions. Depress Anxiety. 2010;27(9):791-8.

12. Connor KM, Davidson JR, Hughes DC, Swartz MS, Blazer DG, George LK. The impact of borderline personality disorder on post-traumatic stress in the community: a study of health status, health utilization, and functioning. Compr Psychiatry. 2002;43(1):41-8.

13. Heffernan K, Cloitre M. A comparison of posttraumatic stress disorder with and without borderline personality disorder among women with a history of childhood sexual abuse: etiological and clinical characteristics. J Nerv Ment Dis. 2000;188(9):589-95.

14. Marshall-Berenz EC, Morrison JA, Schumacher JA, Coffey SF. Affect intensity and lability: the role of posttraumatic stress disorder symptoms in borderline personality disorder. Depress Anxiety. 2011;28(5):393-9.

15. Zlotnick C, Johnson DM, Yen S, Battle CL, Sanislow $\mathrm{CA}$, Skodol AE, et al. Clinical features and impairment in women with Borderline Personality Disorder (BPD) with Posttraumatic Stress Disorder (PTSD), BPD without PTSD, and other personality disorders with PTSD. J Nerv Ment Dis. 2003;191(11):706-13.

16. Zanarini MC, Frankenburg FR, Hennen J, Reich DB, Silk KR. Axis I comorbidity in patients with borderline personality disorder: 6-year follow-up and prediction of time to remission. Am J Psychiatry. 2004;161(11):2108-14.

17. Zanarini MC, Frankenburg FR, Hennen J, Reich DB, Silk KR. Prediction of the 10-year course of borderline personality disorder. Am J Psychiatr. 2006;163(5):827-32.

18. Cloitre M, Stovall-McClough KC, Nooner K, Zorbas P, Cherry S, Jackson CL, et al. Treatment for PTSD related to childhood abuse: a randomized controlled trial. Am J Psychiatry. 2010;167(8):915-24.

19. Mueser KT, Rosenberg SD, Xie H, Jankowski MK, Bolton $\mathrm{EE}, \mathrm{Lu} \mathrm{W}$, et al. A randomized controlled trial of cognitive-behavioral treatment for posttraumatic stress disorder in severe mental illness. J Consult Clin Psychol. 2008;76(2):259-71.

20. Sachsse U, Vogel C, Leichsenring F. Results of psychodynamically oriented trauma-focused inpatient treatment for women with complex posttraumatic stress 
disorder (PTSD) and borderline personality disorder (BPD). Bull Menninger Clin. 2006;70(2):125-44.

21. Foa E, Hembree E, Rothbaum B. Prolonged Exposure Therapy for PTSD: emotional processing of traumatic experiences. New York: Oxford University Press; 2007.

22. Powers MB, Halpern JM, Ferenschak MP, Gillihan SJ, Foa EB. A meta-analytic review of prolonged exposure for posttraumatic stress disorder. Clin Psychol Rev. 2010;30(6):635-41.

23. Feeny NC, Zoellner LA, Foa EB. Treatment outcome for chronic PTSD among female assault victims with borderline personality characteristics: a preliminary examination. J Personal Disord. 2002;16(1):30-40.

24. Clarke SB, Rizvi S, Resick PA. Borderline personality characteristics and treatment outcome in cognitivebehavioral treatments for PTSD in female rape victims. Behav Ther. 2008;39:72-8.

25. van Minnen A, Harned MS, Zoellner LA, Mills K. Examining potential contraindications for Prolonged Exposure therapy for PTSD. European Journal of Psychotraumatology 2012; 3. doi:10.3402/ ejpt.v3i0.18805-PMCID.

26. Resick PA, Schnicke MK. Cognitive processing therapy for rape victims: a treatment manual. Newbury Park: Sage; 1993.

27. Chard KM, Ricksecker EG, Healy ET, Karlin BE, Resick PA. Dissemination and experience with cognitive processing therapy. J Rehabil Res Dev. 2012;49(5):66778.

28.• Bohus M, Dyer AS, Priebe K, Kruger A, Kleindienst N, Schmahl C, et al. Dialectical behaviour therapy for post-traumatic stress disorder after childhood sexual abuse in patients with and without borderline personality disorder: a randomised controlled trial.

Psychother Psychosom. 2013;82(4):221-33.

This is the first RCT of DBT-PTSD, a 12-week residential, phasebased treatment that combines strategies from DBT and trauma-focused treatments for women with PTSD related to childhood sexual abuse. Results indicate that DBT-PTSD was superior to a treatment as usual - waitlist control and it was equally effective for women with and without BPD.

29. Steil R, Dyer A, Priebe K, Kleindienst N, Bohus M. Dialectical behavior therapy for posttraumatic stress disorder related to childhood sexual abuse: a pilot study of an intensive residential treatment program. J Trauma Stress. 2011;24(1):102-6.

30. Linehan MM. Cognitive-behavioral treatment of borderline personality disorder. New York: Guilford Press; 1993.

31.• Harned MS, Korslund KE, Linehan MM. A pilot randomized controlled trial of dialectical behavior therapy with and without the dialectical behavior therapy prolonged exposure protocol for suicidal and selfinjuring women with borderline personality disorder and PTSD. Behav Res Ther. 2014;55:7-17.

This is the first RCT of DBT + DBT PE, a 1-year outpatient, integrated treatment for women with BPD, PTSD, and recent and recurrent suicidal and self-injurious behavior. Results indicate that DBT + DBT PE had larger effects on PTSD and other outcomes than DBT alone and the two treatments were comparable in terms of safety and acceptability.

32. Harned MS, Jackson SC, Comtois KA, Linehan MM. Dialectical behavior therapy as a precursor to PTSD treatment for suicidal and/or self-injuring women with borderline personality disorder. J Trauma Stress. 2010;23(4):421-9.

33. Barnicot K, Priebe S. Post-traumatic stress disorder and the outcome of dialectical behaviour therapy for borderline personality disorder. Personal Ment Health. 2013;7(3):181-90.

34. Harned MS. Treatment of posttraumatic stress disorder with comorbid borderline personality disorder. In: McKay D, Storch E, editors. Handbook of treating variants and complications in anxiety disorders. New York: Springer Press; 2013.

35. Harned MS, Linehan MM. Integrating dialectical behavior therapy and prolonged exposure to treat co-occurring borderline personality disorder and PTSD: two case studies. Cogn Behav Pract. 2008;15(3):263-76.

36. Harned MS, Korslund KE, Foa EB, Linehan MM. Treating PTSD in suicidal and self-injuring women with borderline personality disorder: development and preliminary evaluation of a Dialectical Behavior Therapy Prolonged Exposure Protocol. Behav Res Ther. 2012;50(6):381-6.

This is the initial open feasibility trial evaluating DBT + DBT PE for women with BPD, PTSD, and recent and recurrent suicidal and self-injurious behavior. The results provide preliminary evidence that this integrated treatment is safe and acceptable to patients who exhibited large and significant pre-post reductions in PTSD and other outcomes.

37. Schauer M, Neuner F, Elbert T. Narrative exposure therapy: a short-term intervention for traumatic stress disorders. 2nd ed. Cambridge: Hogrefe Publishing; 2011.

38. Pabst A, Schauer M, Bernhardt K, Ruf M, Goder R, Rosentraeger $\mathrm{R}$, et al. Treatment of patients with borderline personality disorder and comorbid posttraumatic stress disorder using narrative exposure therapy: a feasibility study. Psychother Psychosom. 2012;81(1):61-3.

39. Foa EB, McLean CP, Capaldi S, Rosenfield D. Prolonged exposure vs supportive counseling for sexual abuse-related PTSD in adolescent girls: a randomized clinical trial. JAMA. 2013;310(24):2650-7.

40. Gilboa-Schechtman E, Foa EB, Shafran N, Aderka IM, Powers MB, Rachamim L, et al. Prolonged exposure versus dynamic therapy for adolescent PTSD: a pilot randomized controlled trial. J Am Acad Child Adolesc Psychiatry. 2010;49(10):1034-42.

41. Ruf M, Schauer M, Neuner F, Catani C, Schauer E, Elbert T. Narrative exposure therapy for 7- to 16-yearolds: a randomized controlled trial with traumatized refugee children. J Trauma Stress. 2010;23(4):437-45.

42. Mehlum L, Tormoen AJ, Ramberg M, Haga E, Diep LM, Laberg S et al. Dialectical Behavior Therapy for adolescents with repeated suicidal and self-harming behavior: A randomized trial. J Am Acad Child Adolesc Psychiatry. In press. 